\title{
The reproductive inhibitory effects of levonorgestrel, quinestrol, and EP-1 in Brandt's vole (Lasiopodomys brandtii)
}

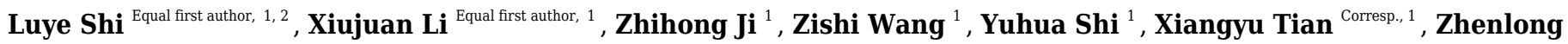 \\ Wang ${ }^{\text {Corresp. } 1}$ \\ ${ }^{1}$ School of Life Sciences, Zhengzhou University, Zhengzhou, Henan, China \\ 2 School of Physical Education (Main campus), Zhengzhou University, Zhengzhou, Henan, China \\ Corresponding Authors: Xiangyu Tian, Zhenlong Wang \\ Email address: 201531200038@mail.bnu.edu.cn, wzl@zzu.edu.cn
}

Background. Rodent pests can inflict devastating impacts on agriculture and the environment, leading to significant economic damage associated with their high species diversity, reproductive rates, and adaptability. Fertility control methods can indirectly control rodent pest populations as well as limit ecological consequences and environmental concerns caused by lethal chemical poisons. Brandt's voles, which are common rodent pests that are found in the grasslands of middle-eastern Inner Mongolia, eastern regions of Mongolia, and some regions of southern Russia, were assessed in the present study.

Methods. We evaluated the effects of levonorgestrel, quinestrol, and a 1:1 mixture of the two (EP-1) on reproductive behavior as well as changes in the reproductive system, reproductive hormone levels, and toxicity in Brandt's voles.

Results. Our results revealed that all three fertility control agents can cause sterility at a dosage of 2 $\mathrm{mg} / \mathrm{kg}$. However, quinestrol caused a greater degree of toxicity, as determined by visible liver damage and reduced expression of the detoxifying molecule CYP1A2. Of the remaining two fertility control agents, EP-1 was superior to levonorgestrel in inhibiting the secretion of follicle-stimulating hormone and causing sterility. We believe that these findings will help promote the use of these fertility control agents and, in turn, reduce the use of chemical poisons and limit their detrimental ecological and environmental impacts. 


\section{The reproductive inhibitory effects of levonorgestrel,}

\section{2 quinestrol, and EP-1 in Brandt's vole (Lasiopodomys}

\section{3 brandtii)}

4 Luye Shi ${ }^{1,2 \dagger}$, Xiujuan $\mathrm{Li}^{1 \dagger}$, Zhihong $\mathrm{Ji}^{1}$, Zishi Wang ${ }^{1}$, Yuhua Shi ${ }^{1}$, Xiangyu Tian ${ }^{1 *}$ and Zhenlong

5 Wang $^{1 *}$

6

$7 \quad{ }^{1}$ School of Life Sciences, Zhengzhou University, Zhengzhou 450001, Henan, China

$8 \quad{ }^{2}$ School of Physical Education (Main campus), Zhengzhou University, Zhengzhou 450001,

9 Henan, China

$11 \dagger$ Luye Shi and Xiujuan Li contributed equally to this work.

12 Corresponding Author:

13 School of Life Sciences, Zhengzhou University, NO.101 Kexue Road, High-Tech Development

14 Zone Of States, 450001, Zhengzhou, P.R.China

15 Xiangyu Tian, Email address: 201531200038@mail.bnu.edu.cn

16 Zhenlong Wang, Email address: wzl@zzu.edu.cn

\section{Abstract}

Background. Rodent pests can inflict devastating impacts on agriculture and the environment,

leading to significant economic damage associated with their high species diversity, reproductive rates, and adaptability. Fertility control methods could indirectly control rodent pest populations as well as limit ecological consequences and environmental concerns caused by lethal chemical poisons. Brandt's voles, which are common rodent pests found in the grasslands of middleeastern Inner Mongolia, eastern regions of Mongolia, and some regions of southern Russia, were assessed in the present study.

Methods. We evaluated the effects of a $2-\mathrm{mg} / \mathrm{kg}$ dose of levonorgestrel and quinestrol and a 1:1 mixture of the two (EP-1) on reproductive behavior as well as changes in the reproductive system, reproductive hormone levels, and toxicity in Brandt's voles.

Results. Our results revealed that all three fertility control agents can cause reproductive 
inhibition at a dosage of $2 \mathrm{mg} / \mathrm{kg}$. However, quinestrol caused a greater degree of toxicity, as determined by visible liver damage and reduced expression of the detoxifying molecule CYP1A2. Of the remaining two fertility control agents, EP-1 was superior to levonorgestrel in inhibiting the secretion of follicle-stimulating hormone and causing reproductive inhibition. We believe that these findings could help promote the use of these fertility control agents and, in turn, reduce the use of chemical poisons and limit their detrimental ecological and environmental impacts.

Key words: Brandt's voles (Lasiopodomys brandtii), reproductive inhibition, levonorgestrel, quinestrol, EP-1

\section{Introduction}

Rodents are highly diverse, adaptable, and have high reproductive rates (REF); such characteristics mean that several species of rodents are considered major pests because they cause devastating economic impacts to agriculture and the environment and are involved in spreading numerous diseases to humans and livestock (Bordes et al. 2015; Capizzi et al. 2014; Heroldová et al. 2012; Meerburg et al. 2009; Singleton 2003; Singleton et al. 1999; Stenseth et al. 2003). Besides, rodents hinder the attempts to alleviate poverty in Africa (Mwanjabe et al. 2002) and Asia (Singleton 2003). In Asia, a loss of 5\% of total rice production, amounting to 30 million tons (sufficient to feed 180 million individuals for 12 months), has been reported (Singleton 2003). Previous rodent management studies have primarily focused on mechanical controls (Mayamba et al., 2019), chemical rodenticides (Goulois et al., 2017), and biological controls (Mayamba et al. 2019; Stenseth et al. 2003). However, these prevention control measures can lead to ecological threats of their own (Ettlin \& Prentice 2002). In recent years, the Integrated Pest Management (IPM) and Ecologically Based Rodent Management (EBRM) strategies, which are based on ecological understanding, agronomy, environmental awareness, and sociocultural considerations, have increasingly been accepted by individuals (Altieri et al. 1983; Krijger et al. 2017; Singleton et al. 1999). Among the existing IPM and EBRM rodent control strategies, fertility control has been identified as a more appropriate, long-term control strategy compared with death by chemical poisoning because it is environmentally benign and humane (Chambers et al. 1999; Liu et al. 2013; Shi et al. 2002; Tang et al. 2012a; Tang et al. 2012b; Zhang 2000). This approach is consistent with the concept of non-toxic residues in EBRM strategy and can 
61 regulate population density by influencing the birth rate of pups (Dell'Omo \& Palmery 2002; Fu 62 et al. 2013; Liu et al. 2013; Liu 2019; Su et al. 2016).

Steroid hormone can cause infertility among animals by interfering with the normal functioning of the hypothalamic-pituitary-gonadal axis (Chen et al. 2017; Massawe et al. 2018; Su et al. 2016; Su et al. 2019; Su et al. 2015; Zhang 2004). Both levonorgestrel (L), a synthetic form of progesterone, and quinestrol (Q), a synthetic estrogen, cause varying degrees of reproductive inhibition in a variety of rodents when combined (EP-1) (Massawe et al. 2018; Zhang 2004). In recent years, research regarding the combination of the two agents in rodent pests has revealed the molecular mechanism associated with infertility, its environmental safety, and appropriate dosage (Liu et al. 2012; Wang et al. 2011). Studies have shown that EP-1 reduces follicle-stimulating hormone levels and promotes luteinizing hormone levels, thereby fertility (Lv \& Shi 2011; Lv \& Shi 2012). As a fertility control agent, EP-1 has the advantages of high reproductive inhibition, good palatability, and strong sustainability. Moreover, it rapidly degrades and does not cause excessive environmental destruction (Tang et al. 2012a; Tang et al. 2012b).

Although fertility control agents, such as oestrogen and triptolide, can cause temporary infertility in animals, they can be toxic (Kejuan et al. 2007; Lipschutz et al. 1966; Maier \& Herman 2001; van Aerts et al. 2019; Xu et al. 2019; Yuan et al. 2019) and can cause death at higher dosages (Lehmann et al. 1989; Zhang et al. 2015; Zhang 2015). Therefore, it is important to estimate the toxicity in animals prior to using fertility control agents (Ettlin \& Prentice 2002; Gao \& Short 1993; Ratti et al. 2015; Turner et al. 2011; Zhang et al. 2015). CYP1A2 is a member of the cytochrome P450 family that is involved in the metabolism of numerous drugs. In addition, it is responsible for the activation of precursors and carcinogens in the body (Anttila et al. 2011; Jiang et al. 2010; Zanger \& Schwab 2013). Several factors, such as aging, genetics, diet, disease, and toxic substances, can affect the metabolic activity of P450 enzymes (Stavropoulou et al. 2018; Zanger \& Schwab 2013). Ingestion of drugs, can induce various transformations due to the action of microsomal cytochrome P450 and glucuronide transferase (Jiang et al. 2010; Stavropoulou et al. 2018). Therefore, as a detoxifying molecule, the activity of CYP1A2 may indicate toxicity levels in the body.

Brandt's voles (Lasiopodomys brandtii) are native to the grasslands of middle-eastern Inner 
92 Mongolia, eastern regions of Mongolia, and some regions of southern Russia (Li et al. 2017).

93 They are considered pests because they can cause serious damage to grassland vegetation and grazing crops and can spread disease (Zhang et al. 2003). In the present study, Brandt's voles were treated with levonorgestrel, quinestrol, and a 1:1 mixture (EP-1) at a dosage of $2 \mathrm{mg} / \mathrm{kg}$ to determine the effects of each agent on reproductive status as well as their toxicity. During the experiment, the body weight of voles in different treatment groups was measured once every 7 days, and the animals were killed on Day 30 from the commencement of the treatment for measuring the effect of the steroids on the reproductive system and reproductive hormones and CYP1A2 levels in different treatment groups.

\section{Materials \& Methods}

\section{Animal materials and experimental groups}

Adult Brandt's voles were obtained from the Chinese Academy of Agricultural Science and were maintained in individual polycarbonate cages $(37 \times 26 \times 17 \mathrm{~cm})$ on a $14-\mathrm{h} / 10$-h light/dark cycle at $20^{\circ} \mathrm{C}-24^{\circ} \mathrm{C}$ and $35 \%-50 \%$ humidity for at least 1 month. During the feeding period, voles were regularly fed with rat and rabbit feed (produced by the Henan experimental animal center, Zhengzhou, China) and fresh carrots.

To test the effect of various fertility control agents, 56 six-month-old healthy adult voles weighing between 40 and 55 g were randomly divided into four groups $(n=14$ in each group; 7 males, 7 females) as follows: L group (levonorgestrel), Q group (quinestrol), EP-1 group (1:1 mixture of levonorgestrel and quinestrol), and a control group (no fertility control agent; Table S1).

The experimental protocol was approved by the Animal Care and Use Committee of Zhengzhou University and was conducted in accordance with the Guide for the Care and Use of Laboratory Animals of China.

\section{Fertility control agent preparation and animal treatment}

Levonorgestrel, quinestrol, and their 1:1 mixture (Dalian Meilun Biotechnology Co., Ltd., China) were dissolved in $0.2 \mathrm{~g} / \mathrm{mL}$ 2-hydroxypropyl- $\beta$-cyclodextrin (HPCD, Shandong Binzhou Zhiyuan Biotechnology Co., Ltd., China). The final concentrations of the three fertility control agents were $0.5 \mathrm{mg} / \mathrm{mL}$, and they were stored at $4^{\circ} \mathrm{C}$ until required. 
The treatment with the fertility control agents was performed for 7 days. To avoid the effects of different circadian rhythms on the animals, all experiments were conducted in the morning. During the experiment, animals in different treatment groups were orally gavaged with different fertility control agents at a dosage of $2 \mathrm{mg} / \mathrm{kg}$ using an elbow gavage needle (No. 12 elbow gavage needle, Beijing Zhe Cheng Technology Co., Ltd.) at 9:00 a.m. daily. The dosage was based on previous studies by Wang and Su (Wang et al. 2011; Su et al. 2019) where the dosage of levonorgestrel and quinestrol was $2 \mathrm{mg} / \mathrm{kg}$ and the dosage of EP-1 was $1 \mathrm{mg} / \mathrm{kg}$ of levonorgestrel and $1 \mathrm{mg} / \mathrm{kg}$ of quinestrol. Each experimental animal was separately housed during treatments, following which animals in each treatment group were housed in male-female pairs.

\section{Measurements of body weight, pregnancy rate, and number of fetuses}

The weight of the experimental animals was measured five times: before injection with the fertility control agents and on days $7,14,21$, and 28 from the commencement of the treatment.

All animals were killed 30 days after the start of treatment with an overdose of pentobarbital sodium $(1 \%, 1 \mathrm{~mL})$; female individuals in each group were dissected, and the pregnancy rate, number of fetuses, and rate of fetal malformation were determined.

\section{Paraffin sections of the reproductive system and liver tissue}

After the animals were killed, the testes and epididymides were removed from the male voles and the uteri and ovaries were removed from the female voles. Further, liver tissues of each animal were removed. Each organ was sequentially weighed and fixed in buffered paraformaldehyde (4\%) for $24 \mathrm{~h}$.

Paraffin sections of each reproductive organ and liver tissues were sequentially prepared and stained with hematoxylin and eosin, and the prepared sections were assessed using a microscope with a magnification of $\times 100$. Five fields of view were randomly selected for histological observation and photographing.

\section{Determination of estradiol, follicle-stimulating hormone, testosterone, and corticosterone} levels 
Sub-orbital sinus blood was collected on Day 30. The blood was stored at room temperature for 20-30 min to clot, and serum was extracted from the blood via centrifugation (3000 rpm, 20 $\min$ ) and was transferred into a $-20^{\circ} \mathrm{C}$ freezer. The serum levels of estradiol, follicle-stimulating hormone, testosterone, and corticosterone were measured using the E2 ELISA kit, FSH ELISA kit, T ELISA kit, and CORT ELISA kit (Shanghai MLBIO Biotechnology Co., Ltd., China), respectively. All procedures were performed in accordance with the manufacturer's instructions.

\section{Total RNA extraction and quantitative real-time PCR}

Total RNA was extracted from the brain tissue of each animal using TRIzol reagent (Invitrogen, Carlsbad, CA, USA), in accordance with the manufacturer's instructions. Residual DNA was removed by treatment with RNase-free DNase I (Takara Bio, Dalian, China). RNA integrity was verified using agarose gel electrophoresis (1.2\%), and RNA concentrations were measured using an Agilent 2100 Bioanalyzer (Agilent Technologies, Santa Clara, CA, USA).

The total RNA extracted from the brain tissues was reverse-transcribed into cDNA using a reverse transcription kit (Takara Bio, Japan). The obtained cDNA was stored at $-20^{\circ} \mathrm{C}$ prior to real-time PCR.

The CYP1A2 sequences of the near relatives of Brandt's voles were determined and compared on National Center for Biotechnology Information before designing primers for realtime PCR using Primer3 online software (Untergasser et al. 2012). The primers were further verified via sequencing after the PCR products were assembled into cloning vectors. Details of primer sequences are shown in Table 1.

Real-time PCR was performed on a Rotor-Gene 3000 fluorescence quantitative PCR instrument (Corbett Research, Germany) using a default reaction procedure configuration. The reaction volume was $20 \mu \mathrm{L}$ and included $0.5 \mu \mathrm{L}$ each of specific forward and reverse primers $(20$ $\mu \mathrm{M}), 2 \mu \mathrm{L}$ of the diluted cDNA template, $1 \mu \mathrm{L}$ of the PrimeScript RT Enzyme Mix, $6 \mu \mathrm{L}$ of the $5 \times$ PrimeScript Buffer, and $10 \mu \mathrm{L}$ of RNase-free $\mathrm{H}_{2} \mathrm{O}$. $\beta$-actin was used as the internal control gene (forward: GTCGTACCACTGGCATTGTG; reverse: CCATCTCTTGCTCGAAGTCC), and the relative gene expression was determined using the comparative CT method (Pfaffl 2001).

\section{Statistics analysis}


All data were analyzed using SPSS (version 22.0, SPSS Inc., USA) and presented as mean \pm SE. A p value of $<0.05$ denoted statistical significance. A generalized linear model with repeated measurements was used to analyze the rate of change of body weight, and two-way ANOVA was used to analyze the effects of the fertility control agents on the reproductive system, hormone levels, and gene expression level in each group.

\section{Results}

\section{Effects of fertility control agents on the body weight}

During the experiment, the body weight of the L treatment group increased during the first 21 days following which it began to slowly decrease. The remaining three treatment groups (control, Q, and EP-1) initially showed decreases in body weight but subsequently increased (Fig. 1; Table S1). The maximum weight loss in the Q group occurred by Day 14 of the experiment, and the weight was significantly lower in this group than that in the other three groups ( $p=$ 0.031). This indicated that quinestrol significantly reduced the body weight of Brandt's voles $(p$ $=0.011)$ but levonorgestrel and EP-1 did not $(p=0.082, p=0.113$, respectively).

\section{Effects of fertility control agents on reproduction}

On Day 30 of the experiment, 6 of the 7 females in the control group were pregnant, with a pregnancy rate of $85.7 \%$. However, among the three treated groups, only one female in the L group experienced a failed pregnancy, and none of the other females showed any signs of pregnancy (Table 2). All embryos in the control group showed normal development, whereas the two embryos in the L group were both resorbed fetuses.

\section{Effects of fertility control agents on the reproductive system}

Compared with the control group, L $(p=0.03), \mathrm{Q}(p=0.02)$, and EP-1 $(p=0.02)$ demonstrated a significantly reduced uterine weight in the female voles (Fig. 2A). Furthermore, the results of the morphological observations showed that the uterine walls in females in the three treatment groups were thinner than those in females in the control group (Figure S1). However, the three fertility control agents had no significant effect on the ovarian weight of the female voles ( $p=0.81$ for L, $p=0.93$ for Q, and $p=0.79$ for EP-1; Fig. 2B). In addition, no significant difference was observed in the development of ovarian tissue in the treatment groups 
215 (Figure S2).

216 Compared with the males in the control group, the testicular shape and volume in males in

217 the L and EP-1 groups did not show significant changes, whereas the testes of a few males in the

218 Q group showed a darker color and decreased volume (Figure S3). In addition, compared with

219 the other three groups, males in the Q group showed a significant decrease in their testicular

220 weight by approximately $40 \%$ ( $p=0.03$, Fig. 2 C), a sparser seminiferous tubule, acute apoptosis

221 in sperm cells, and testicular stromal and supporting cells at all levels (Figure S4). Similar to the

222 findings from the morphological observations, compared with the control group, the epididymal

223 weight of the individuals in the Q group decreased by $50.6 \%$, with extremely few individuals

224 experiencing unilateral epididymal atrophy ( $p=0.01$, Fig. 2D).

225

226

227

228

229

230

231

232

233

234

235

236

237

238

239

240

241

242

243

244

245

\section{Effects of fertility control agents on the reproductive hormones}

No significant differences were observed in estradiol, testosterone, or corticosterone levels in the animals among different treatment groups (Fig. 3). However, the follicle-stimulating hormone levels in the L $(p=0.03)$ and EP-1 $(p=0.01)$ groups were significantly lower than those in the control group (Fig. 3).

\section{Effects of fertility control agents on the expression of CYP1A2}

Compared with the control group, the expression of CYP1A2 in the other three groups was significantly reduced. Specifically, the expression levels of CYP1A2 in the L, Q, and EP-1 groups were reduced by $40 \%, 61 \%$, and $43 \%$, respectively (Fig. 4A).

\section{Effects of fertility control agents on liver tissue}

Following treatment, the liver tissue in animals treated with quinestrol appeared dark in color and lost its normal toughness. Furthermore, a small number of EP-1-treated animals showed the abovementioned liver morphological changes, whereas no liver morphological changes were observed in the L or control groups.

From the liver tissue sections, the hepatocytes of the control, L, and EP-1 groups showed normal development, whereas those of the Q group showed tissue edema and local bleeding (Fig. 4B). 


\section{Discussion}

247 Currently, chemical poisoning using agents such as anticoagulant rodenticides and zinc

248 phosphide remains one of the most important methods for rodent pest control (Haim et al. 2007;

249 Labuschagne et al. 2016; Liu 2019). EBRM has recently been implemented in several countries,

250 such as Vietnam and Indonesia as well as in the African continent, and mainly involves the use

251 of trap-barrier systems, community rat hunting campaigns, and habitat management (Brown et al.

252 2005; Brown \& Khamphoukeo 2007; Jacob et al. 2008; Massawe et al. 2011; Singleton et al.

253 2005; Wang et al. 2017). The addition of fertility control as an ecologically friendly method has

254 not yet been widely possible. In the present study, we evaluated the reproductive inhibitory effect

255 of quinestrol, levonorgestrel, and EP-1 in Brandt's voles. Our findings suggest that the effects of

256 these fertility control agents require further research so that they can be applied as part of an

257 integrated approach in future large-scale applications.

258 The study results showed that quinestrol treatment led to significantly reduced body weight

259 of voles; however, levonorgestrel and EP-1 treatment had no significant effects, suggesting that

260 quinestrol is more toxic than the remaining two fertility control agents. Appropriate fertility

261 control agents should cause competitive reproduction interference without affecting the animal's

262 normal development and other biological functions. Therefore, any treatment administered

263 should avoid killing the pests, and the doses should not negatively affect their growth and

264 development (Liu et al. 2018; Turner et al. 2011). In previous studies, Su et al. found that EP-1

265 in different ratios $(1: 1,2: 1,1: 2)$ can reduce the sperm density and vitality of male mice and

266 cause atrophy of the epididymis and seminal vesicles; on the other hand, the 1: 1 ratio of EP-1

267 had the best effect on reproductive inhibition (Su et al. 2019). In addition, Wang et al. proved

268 that $2 \mathrm{mg} / \mathrm{kg}$ of quinestrol had the best reproductive inhibition effect on Brandt 's voles by

269 comparing different concentrations of infertility reproductive inhibitory effects and could sustain

270 for more than 90 days (Wang et al. 2015). In our study, we referred to these experiences to select

271 the reproduction inhibitor dose and EP-1 ratio. The results showed that a dose concentration of 2

$272 \mathrm{mg} / \mathrm{kg}$ and EP-1 ratio of 1: 1 were both appropriate, significantly inhibiting the reproductive

273 process of Brandt 's voles without affecting the normal developmental of animals. However,

274 levonorgestrel and EP-1 appeared less toxic to the animals than quinestrol.

275 The reproductive behavior analysis indicated that all three fertility control agents

276 successfully sterilized the Brandt's voles. The uterine weight (g/100 g body weight) of females 
277 treated with all three fertility control agents was significantly lower than that of the females in

278 the control group, suggesting that the three fertility control agents had inhibited reproduction. In

279 addition to the effect on the uterine weight, the testicular and epididymal weight ( $\mathrm{g} / 100 \mathrm{~g}$ body

280 weight) of the males in the Q group were significantly lower than those in the control group,

281 suggesting that quinestrol inhibits reproduction in both female and male Brandt's vole

282 individuals. However, although the uterine walls of the females in the control group were thinner

283 than those in the control group, this may be due to the fact that the females in the control group

284 experienced pregnancy, so the thickness of the uterine wall could not be used to judge the effect

285 of the sterility agents. Previous research has shown that quinestrol is a stable estradiol

286 homologue that is stored in the adipose tissue and can be slowly released (Zhao et al. 2007).

287 Quinestrol primarily inhibits the release of gonadotropin releasing hormone from the

288 hypothalamus, thereby inhibiting follicle growth, whereas levonorgestrel primarily inhibits the

289 release of follicle-stimulating hormone from the pituitary gland to block ovulation and disrupts

290 the proliferation and differentiation of endometrial cells, preventing embryonic implantation

291 (Zhao et al. 2007).

292 Previous studies in mice and humans have shown that fertility control agents can inhibit

293 pregnancy by interfering with the synthesis of follicle-stimulating hormone, estradiol,

294 testosterone, corticosterone, and other reproductive hormones (Spona \& Huber 1987). In the

295 present study, 30 days after the commencement of fertility control agent treatments, no

296 significant difference was observed in the estradiol, testosterone, or corticosterone hormone

297 levels. However, we observed a significant decrease in follicle-stimulating hormone levels in the

298 EP-1-treated group; this phenomenon can be attributed to mixed levonorgestrel and quinestrol

299 interfering with the in vivo regulation of the hypothalamic-pituitary-adrenal axis (Bednarek

300 2007; Likis 2002). However, the specific interference mechanism of EP-1 and why

301 levonorgestrel and quinestrol do not inhibit follicle-stimulating hormone levels when used alone

302 still require further research.

303 CYP1A2 is a member of the cytochrome P450 enzyme system, which mediates over $90 \%$ of 304 the drug transformation process in vivo (Porter \& Coon 1991). Recently, research on CYP1A2305 mediated drug metabolism has shown that several drugs can cause toxic side effects on the body 306 by inhibiting the activity of CYP1A2 (Faber et al. 2005; Huang et al. 2014; Johnson et al. 2012; 307 Li et al. 2018; Martinez et al. 2013; Wei et al. 2018). We observed that all three fertility control 
agents inhibited the expression of CYP1A2 in Brandt's voles. Among the three fertility control agents, the strongest inhibitory effect was observed in the Q group, which was further confirmed by the observation of liver sections, indicating that quinestrol is more toxic than the other two fertility control agents.

In summary, our results show that all three fertility control agents can cause reproductive inhibition in Brandt's voles at a dosage of $2 \mathrm{mg} / \mathrm{kg}$. However, due to the limitation of the number of animals and experimental conditions, we did not pair the animals of the reproductive inhibition treatment groups with healthy and untreated individuals in the matching process after gavage, making it impossible to distinguish whether reproductive inhibition was caused by the female, male, or both in subsequent analysis. Our current findings suggest that EP-1 was superior to levonorgestrel in inhibiting the secretion of follicle-stimulating hormone and causing reproductive inhibition. Furthermore, compared with the other two agents, quinestrol caused a greater degree of toxicity. Therefore, EP-1 administered at a dosage of $2 \mathrm{mg} / \mathrm{kg}$ appears adequate to inhibit the reproduction of Brandt's voles.

\section{Conclusion}

In previous studies, both levonorgestrel and quinestrol were demonstrated to have inhibitory effects on reproduction in various rodents (Huo et al. 2007; Liu et al. 2017; Lv \& Shi 2011; Lv \& Shi 2012; Wan et al. 2006). However, the use of biological fertility control agents for pest control has not yet been widely implemented. One possible reason for this is that specific dosages and side effects have not yet been established. In the present study, we have elaborated the effects of the three fertility control agents on the reproductive system, reproductive hormone levels, CYP1A2 expression level, and liver damage. We determined that EP-1 was the most suitable fertility control agents for the reproductive inhibition of Brandt's voles. We hope that these results will help to promote the field use of these fertility control agents so that they can effectively reduce rodent pest populations as well as limit the ecological damage to the environment associated with chemical poisons.

\section{Acknowledgements}

Besides the funding support, we thank Shiming Gu, Mengke Li, Xuqin Wang, Yue Wu, and 
338

339

340

341

342

343

344

345

346

347

348

349

350

351

352

353

354

355

356

357

358

359

360

361

362

363

364

365

366

367

368

Mengyang Li for their help in feeding the experimental animals.

\section{References}

Altieri MA, Martin P, and Lewis W. 1983. A quest for ecologically based pest management systems. Environmental Management 7:91-99 DOI: 10.1007/BF01867047.

Anttila S, Raunio H, and Hakkola J. 2011. Cytochrome P450-mediated pulmonary metabolism of carcinogens: regulation and cross-talk in lung carcinogenesis. American Journal of Respiratory Cell and Molecular Biology 44:583-590 DOI: 10.1111/mam.12019.

Bednarek P. 2007. Continuous-Use Ethinylestradiol/Levonorgestrel $20 \mu \mathrm{g} / 90 \mu \mathrm{g}$ as an Oral Contraceptive. Drugs 67:2480-2480 DOI: https://doi.org/10.2165/00003495-20076716000011.

Bordes F, Blasdell K, and Morand S. 2015. Transmission ecology of rodent-borne diseases: New frontiers. Integrative zoology 10:424-435 DOI: https://doi.org/10.1111/1749-4877.12149.

Brown PR, Davies MJ, Singleton GR, and Croft JD. 2005. Can farm-management practices reduce the impact of house mouse populations on crops in an irrigated farming system? Wildlife Research 31:597-604 DOI: https://doi.org/10.1071/WR03063.

Brown PR, and Khamphoukeo K. 2007. Farmers' knowledge, attitudes, and practices with respect to rodent management in the upland and lowland farming systems of the Lao People's Democratic Republic. Integrative zoology 2:165-173 DOI: https://doi.org/10.1111/j.1749-4877.2007.00055.x.

Capizzi D, Bertolino S, and Mortelliti A. 2014. Rating the rat: global patterns and research priorities in impacts and management of rodent pests. Mammal Review 44:148-162 DOI: https://doi.org/10.1111/mam.12019.

Chambers LK, Singleton GR, and Hinds L. 1999. Fertility control of wild mouse populations: the effects of hormonal competence and an imposed level of sterility. Wildlife Research 26:579591 DOI: https://doi.org/10.1071/WR98093.

Chen Y, Su Q-Q, and Liu Q-S. 2017. Effects of quinestrol on the vocal behavior of mice during courtship interactions. Physiology \& Behavior 173:216-222 DOI: https://doi.org/10.1016/j.physbeh.2017.02.017

Dell'Omo G, and Palmery M. 2002. Fertility control in vertebrate pest species. Contraception 65:273-275 DOI: 10.1016/S0010-7824(02)00285-8. 
369

370

371

372

373

374

375

376

377

378

Ettlin R, and Prentice D. 2002. Unexpected tumour findings in lifetime rodent bioassay studieswhat to do? Toxicology letters 128:17-33 DOI: https://doi.org/10.1016/S03784274(01)00530-6

Faber MS, Jetter A, and Fuhr U. 2005. Assessment of CYP1A2 activity in clinical practice: why, how, and when? Basic \& Clinical Pharmacology \& Toxicology 97:125-134 DOI: 10.1111/j.1742-7843.2005.pto_973160.x.

Fu H, Zhang J, Shi D, and Wu X. 2013. Effects of levonorgestrel-quinestrol (EP-1) treatment on Mongolian gerbil wild populations: a case study. Integrative zoology 8:277-284 DOI: 10.1111/1749-4877.12018.

Gao Y, and Short R. 1993. The control of rodent populations. Oxford reviews of reproductive biology 15:265

Goulois J, Hascoët C, Dorani K, Besse S, Legros L, Benoit E, and Lattard V. 2017. Study of the efficiency of anticoagulant rodenticides to control Mus musculus domesticus introgressed with Mus spretus Vkorc1. Pest Management Science 73:325-331 DOI: 10.1002/ps.4319.

Haim A, Shanas U, Brandes O, and Gilboa A. 2007. Suggesting the use of integrated methods for vole population management in alfalfa fields. Integrative zoology 2:184-190 DOI: https://doi.org/10.1111/j.1749-4877.2007.00054.x

Heroldová M, Bryja J, Jánová E, Suchomel J, and Homolka M. 2012. Rodent damage to natural and replanted mountain forest regeneration. The Scientific World Journal 2012 DOI: $10.1100 / 2012 / 872536$

Huang Y, Zheng S-1, Xu Z-s, and Hou Y. 2014. Effects of Alismatis rhizome on rat cytochrome P450 enzymes. Pharmaceutical Biology 52:681-687 DOI: 10.3109/13880209.2013.864685.

Huo X, Shi D, Wang D. 2007. Effect of levonorgestrel-quinestrol on fertility of female Mongolia gerbils, Meriones unguiculats. Acta Phytophylacica Sin 34:321-325.

Jacob J, Singleton GR, and Hinds LA. 2008. Fertility control of rodent pests. Wildlife Research 35:487-493 DOI: 10.1071/WR07129.

Jiang W, Wang L, Kondraganti SR, Fazili IS, Couroucli XI, Felix EA, and Moorthy B. 2010. Disruption of the gene for CYP1A2, which is expressed primarily in liver, leads to differential regulation of hepatic and pulmonary mouse CYP1A1 expression and augmented human CYP1A1 transcriptional activation in response to 3-methylcholanthrene in vivo. Journal of Pharmacology and Experimental Therapeutics 335:369-379 DOI: 
10.1124/jpet.110.171173.

Johnson CH, Patterson AD, Idle JR, and Gonzalez FJ. 2012. Xenobiotic metabolomics: major impact on the metabolome. Annual Review of Pharmacology and Toxicology 52:37-56 DOI: 10.1146/annurev-pharmtox-010611-134748.

Kejuan F, Meirik O, Yongang D, Yan C, Weijin Z, and Fajans P. 2007. Once-a-month contraceptive pills in China: a review of available evidence. Contraception 75:337-343

Krijger IM, Belmain SR, Singleton GR, Groot Koerkamp PW, and Meerburg BG. 2017. The need to implement the landscape of fear within rodent pest management strategies. Pest Management Science 73:2397-2402 DOI: 10.1002/ps.4626.

Labuschagne L, Swanepoel LH, Taylor PJ, Belmain SR, and Keith M. 2016. Are avian predators effective biological control agents for rodent pest management in agricultural systems? Biological Control 101:94-102 DOI: 10.1016/j.biocontrol.2016.07.003.

Lehmann M, Putz B, Poggel H, and Gunzel P. 1989. Experimental toxicity studies with contraceptive steroids and their relevance for human risk estimation. Advances in applied toxicology. Taylor and Francis London 51-79

Li K, Kohn MH, Zhang S, Wan X, Shi D, and Wang D. 2017. The colonization and divergence patterns of Brandt's vole (Lasiopodomys brandtii) populations reveal evidence of genetic surfing. BMC Evolutionary Biology 17:145 DOI: 10.1186/s12862-017-0995-y.

Li Y, Zhao C, Lu X, Ai X, and Qiu J. 2018. Identification of a cytochrome P450 gene in the earthworm Eisenia fetida and its mRNA expression under enrofloxacin stress. Ecotoxicology and Environmental Safety 150:70-75 DOI: 10.1016/j.ecoenv.2017.12.020.

Likis FE. 2002. Contraceptive applications of estrogen. Journal of midwifery \& women's health 47:139-156 DOI: https://doi.org/10.1016/S1526-9523(02)00234-9.

Lipschutz A, Iglesias R, Salinas S, and Panasevich VI. 1966. Experimental conditions under which contraceptive steroids may become toxic. Nature 212:686-688

Liu H, Zhang G, Wang J, Li J, Song Y, Qiao L, Niu N, Wang J, Ma S, and Li L. 2018. Chemical hybridizing agent SQ-1-induced male sterility in Triticum aestivum L.: a comparative analysis of the anther proteome. BMC Plant Biology 18:7 DOI: 10.1186/s12870-017-1225-X.

Liu M, Luo R, Wang H, Cao G, and Wang Y. 2017. Recovery of fertility in quinestrol-treated or diethylstilbestrol-treated mice: Implications for rodent management. Integrative zoology 12:250-259 DOI: 10.1111/1749-4877.12236. 
431

432

433

434

435

436

437

438

439

440

Liu M, Wan X, Yin Y, Li Y-x, Sun F, Zhang Z, and Wang Y-1. 2012. Subfertile effects of quinestrol and levonorgestrel in male rats. Reproduction, Fertility and Development 24:297308 DOI: 10.1071/RD10221.

Liu Q, Qin J, Chen Q, Wang D, and Shi D. 2013. Fertility control of Rattus nitidus using quinestrol: effects on reproductive organs and social behavior. Integrative zoology 8:9-17 DOI: https://doi.org/10.1111/j.1749-4877.2012.00296.x.

Liu X-h. 2019. Rodent biology and management: Current status, opinion and challenges in China. Journal of Integrative Agriculture 18:830-839 DOI: https://doi.org/10.1016/S20953119(18)61943-4

Lv X-H, and Shi D-Z. 2011. The effects of quinestrol as a contraceptive in Mongolian gerbils (Meriones unguiculatus). Experimental animals 60:489-496 DOI: 10.1538/expanim.60.489.

Lv X, and Shi D. 2012. Combined effects of levonorgestrel and quinestrol on reproductive hormone levels and receptor expression in females of the Mongolian gerbil (Meriones unguiculatus). Zoological science 29:37-42 DOI: 10.2108/zsj.29.37.

Maier WE, and Herman JR. 2001. Pharmacology and toxicology of ethinyl estradiol and norethindrone acetate in experimental animals. Regulatory Toxicology and Pharmacology 34:53-61 DOI: https://doi.org/10.1006/rtph.2001.1483

Martinez MN, Antonovic L, Court M, Dacasto M, Fink-Gremmels J, Kukanich B, Locuson C, Mealey K, Myers MJ, and Trepanier L. 2013. Challenges in exploring the cytochrome P450 system as a source of variation in canine drug pharmacokinetics. Drug Metabolism Reviews 45:218-230 DOI: 10.3109/03602532.2013.765445.

Massawe AW, Makundi RH, Zhang Z, Mhamphi G, Liu M, Li H-J, and Belmain SR. 2018. Effect of synthetic hormones on reproduction in Mastomys natalensis. Journal of Pest Science 91:157-168 DOI: 10.1007/s10340-017-0894-4.

Massawe AW, Mulungu LS, Makundi RH, Dlamini N, Eiseb SJ, Kirsten F, Mahlaba Ta, Malebane P, Von Maltitz E, and Monadjem A. 2011. Spatial and temporal population dynamics of rodents in three geographically different regions in Africa: implication for ecologically-based rodent management. African Zoology 46:393-405 DOI: https://doi.org/10.1080/15627020.2011.11407513.

Mayamba A, Byamungu RM, Makundi RH, Kimaro DN, Isabirye M, Massawe AW, Kifumba D, Nakiyemba A, Leirs H, and Mdangi ME. 2019. Species composition and community 
462

structure of small pest rodents (Muridae) in cultivated and fallow fields in maize-growing areas in Mayuge district, Eastern Uganda. Ecology and evolution 9:7849-7860 DOI: 10.1002/ece3.5371.

Meerburg BG, Singleton GR, and Kijlstra A. 2009. Rodent-borne diseases and their risks for public health. Critical Reviews in Microbiology 35:221-270 DOI: 10.1080/10408410902989837.

Mwanjabe PS, Sirima FB, and Lusingu J. 2002. Crop losses due to outbreaks of Mastomys natalensis (Smith, 1834) Muridae, Rodentia, in the Lindi Region of Tanzania. International Biodeterioration \& Biodegradation 49:133-137 DOI: https://doi.org/10.1016/S09648305(01)00113-5

Pfaffl MW. 2001. A new mathematical model for relative quantification in real-time RT-PCR. Nucleic Acids Research 29:e45-e45 DOI: https://doi.org/10.1093/nar/29.9.e45

Porter T, and Coon M. 1991. Cytochrome P-450. Multiplicity of isoforms, substrates, and catalytic and regulatory mechanisms. Journal of Biological Chemistry 266:13469-13472

Ratti E, Berry JD, Greenblatt DJ, Loci L, Ellrodt AS, Shefner JM, and Cudkowicz ME. 2015. Preclinical rodent toxicity studies for long term use of ceftriaxone. Toxicology reports 2:1396-1403 DOI: https://doi.org/10.1016/j.toxrep.2015.09.010

Shi D, Wan X, Davis SA, Pech RP, and Zhang Z. 2002. Simulation of lethal control and fertility control in a demographic model for Brandt's vole Microtus brandti. Journal of Applied Ecology 39:337-348 DOI: 10.1016/j.toxrep.2015.09.010.

Singleton G. 2003. Impacts of rodents on rice production in Asia. No. 2169-2019-1613. 2003.

Singleton GR, Jacob J, and Krebs CJ. 2005. Integrated management to reduce rodent damage to lowland rice crops in Indonesia. Agriculture, Ecosystems \& Environment 107:75-82 DOI: https://doi.org/10.1016/j.agee.2004.09.010

Singleton GR, Leirs H, Hinds LA, and Zhang Z. 1999. Ecologically-based management of rodent pests-re-evaluating our approach to an old problem. Ecologically-based Management of Rodent Pests Australian Centre for International Agricultural Research (ACIAR), Canberra $17-29$

Spona J, and Huber J. 1987. Efficacy of low-dose oral contraceptives containing levonorgestrel, gestoden and cyproterone acetate. Gynecologic and Obstetric Investigation 23:184-193 DOI: $10.1159 / 000298860$. 
493

494

495

496

497

498

499

500

501

502

503

504

505

506

507

508

Stavropoulou E, Pircalabioru GG, and Bezirtzoglou E. 2018. The Role of Cytochromes P450 in infection. Frontiers in immunology 9:89 DOI: 10.3389/fimmu.2018.00089.

Stenseth NC, Leirs H, Skonhoft A, Davis SA, Pech RP, Andreassen HP, Singleton GR, Lima M, Machang'u RS, and Makundi RH. 2003. Mice, rats, and people: the bio-economics of agricultural rodent pests. Frontiers in Ecology and the Environment 1:367-375 DOI: 10.1890/1540-9295(2003)001[0367:mraptb]2.0.co;2.

Su Q, Chen Y, Qin J, Wang T, Wang D-H, and Liu Q-S. 2016. Effects of mifepristone and quinestrol on the fertility of female Brandt's voles (Lasiopodomys brandtii) in different reproductive phases. Animal Biology 66:133-143 DOI: 10.1163/15707563-00002492.

Su Q, Chen Y, Qin J, Li H, Liu M, Zhang Z, and Liu Q. 2019. Ratio-dependent effects of quinestrol and levonorgestrel compounds (EP-1) on reproductive parameters of adult male Swiss mice. Pesticide Biochemistry and Physiology 160:181-186 DOI: https://doi.org/10.1016/j.pestbp.2019.08.009

Su Q, Huang XL, Qin J, and Liu QS. 2015. Assessment of effects of mifepristone administration to lactating mice on the development and fertility of their progeny. Journal of Obstetrics and Gynaecology Research 41:575-581

Tang T, Qian K, Shi T, Wang F, Li P, Li J, and Cao Y. 2012a. Photodegradation of quinestrol in waters and the transformation products by UV irradiation. Chemosphere 89:1419-1425 DOI: 10.1016/j.chemosphere.2012.06.011.

Tang T, Shi T, Li D, Xia J, Hu Q, and Cao Y. 2012b. Adsorption properties and degradation dynamics of endocrine-disrupting chemical levonorgestrel in soils. Journal of Agricultural and Food Chemistry 60:3999-4004 DOI: 10.1021/jf300479z.

Turner PV, Brabb T, Pekow C, and Vasbinder MA. 2011. Administration of substances to laboratory animals: routes of administration and factors to consider. Journal of the American Association for Laboratory Animal Science 50:600-613

Untergasser A, Cutcutache I, Koressaar T, Ye J, Faircloth BC, Remm M, and Rozen SG. 2012. Primer3-new capabilities and interfaces. Nucleic Acids Research 40:e115-e115 DOI: 10.1093/nar/gks596.

Van Aerts RM, Bernts LH, Gevers TJ, Kievit W, Koopmans L, Nieboer TE, Nevens F, and Drenth JP. 2019. Estrogen-containing oral contraceptives are associated with polycystic liver disease severity in premenopausal patients. Clinical Pharmacology \& Therapeutics 
106:1338-1345 DOI: 10.1002/cpt.01553

Wan X, Shi Y, Bao X, Guan Q, Yu C, Wang G, Liu W, Zhang Z, Zhong W, and Jiao Y. 2006. Effect of the contraceptive compound (EP--1) on reproduction of the Djungarian hamster (Phodopus campbelli) in the typical steppe. Acta Theriologica Sinica 26:392-397

Wang D, Li N, Liu M, Huang B, Liu Q, and Liu X. 2011. Behavioral evaluation of quinestrol as a sterilant in male Brandt's voles. Physiology \& Behavior 104:1024-1030 DOI: 10.1093/nar/gks596.

Wang D, Li Q, Li K, and Guo Y. 2017. Modified trap barrier system for the management of rodents in maize fields in Jilin Province, China. Crop Protection 98:172-178 DOI: https://doi.org/10.1016/j.cropro.2017.03.025

Wang D, Liu Q, Liu M, Li N, Huang B and Liu X. 2011. Preparation of hydroxypropyl- $\beta$ cyclodextrin inclusion with Levonorgestrel and Quinestrol (EP-1) and its influence on the reproductive organs Brandt's voles (Lasiopodomys brandtii). Acta theriologica sinica 31(1): 79-83 DOI: 10.16829/j.slxb.2011.01.013

Wang T, Guo Y, Wang D, Hai S. 2015. Suppression effects of quinestrol on the reproduction of Brandt's voles (Lasiopodomys brandtii). Acta theriologica sinica 35(1): 87-94

Wei Y-L, Du H-J, Lin Y-P, Wu M-L, and Xu R-a. 2018. Effects of salidroside on rat CYP enzymes by a cocktail of probe drugs. Iranian journal of basic medical sciences 21:422 DOI: 10.22038/IJBMS.2018.26106.6414.

Xu LY, Wu W, Cheng R, Sun LX, Jiang ZZ, Zhang LY, Zhang ZJ, Su YW, and Huang X. 2019. Toxic effects of triptolide on adrenal steroidogenesis in H295R cells and female rats. Journal of Biochemical and Molecular Toxicology 33:e22394 DOI: https://doi.org/10.1002/jbt.22394

Yuan Z, Zhang H, Hasnat M, Ding J, Chen X, Liang P, Sun L, Zhang L, and Jiang Z. 2019. A new perspective of triptolide-associated hepatotoxicity: liver hypersensitivity upon LPS stimulation. Toxicology 414:45-56 DOI: https://doi.org/10.1016/j.tox.2019.01.005

Zanger UM, and Schwab M. 2013. Cytochrome P450 enzymes in drug metabolism: regulation of gene expression, enzyme activities, and impact of genetic variation. Pharmacology \& Therapeutics 138:103-141 DOI: 10.1016/j.pharmthera.2012.12.007.

Zhang Q, Zeng SX, and Lu H. 2015. Determination of maximum tolerated dose and toxicity of Inauhzin in mice. Toxicology reports 2:546-554 DOI: 10.1016/j.toxrep.2015.02.011. 
555 Zhang Z. 2000. Mathematical models of wildlife management by contraception. Ecological 556 Modelling 132:105-113 DOI: https://doi.org/10.1016/S0304-3800(00)00308-2

557 Zhang Z. 2004. Effect of a contraceptive com-pound (EP-1) on fertility of female Brandt's voles, 558 gray hamsters and mid-day gerbils. Acta Zoologica Sinica 50:341-347

559 Zhang Z. 2015. A review on anti-fertility effects of levonorgestrel and quinestrol (EP-1)

560 compounds and its components on small rodents. Acta Theriologica Sinica 35:203-210

561 Zhang Z, Pech R, Davis S, Shi D, Wan X, and Zhong W. 2003. Extrinsic and intrinsic factors 562 determine the eruptive dynamics of Brandt's voles Microtus brandti in Inner Mongolia, 563 China. Oikos 100:299-310 DOI: 10.1034/j.1600-0706.2003.11810.x.

564 Zhao M, Liu M, Li D, Wan X, Hinds LA, Wang Y, and Zhang Z. 2007. Anti-fertility effect of 565 levonorgestrel and quinestrol in Brandt's voles (Lasiopodomys brandtii). Integrative 566 zoology 2:260-268 DOI: https://doi.org/10.1111/j.1749-4877.2007.00059.x. 
569 Figure captions:

570 Figure 1: Body mass (g) of voles in the three fertility control agent-treated groups and 571 control group. The asterisk (*) represents a significant difference from the control group.

572 Figure 2: Uterine weight (A), ovarian weight (B), testicular weight (C), and epididymial 573 weight (D) in the four groups of voles at day 30 of the experiment. Values are presented as $574 \mathrm{~g} / 100 \mathrm{~g}$ body weight and mean $\pm \mathrm{SE}$. The asterisk $\left(^{*}\right)$ represents a significant difference from the 575 control group.

576 Figure 3: Levels of estradiol $(\mathrm{ng} / \mathrm{L}, \mathrm{A})$, follicle-stimulating hormone $(\mu \mathrm{g} / \mathrm{L}, \mathrm{B})$, testosterone $577(\mu \mathrm{g} / \mathrm{L}, \mathrm{C})$, and corticosterone $(\mu \mathrm{g} / \mathrm{L}, \mathrm{D})$ in the four groups of voles on day 30 of the 578 experiment. Each group of data is represented by a box plot. In the group of data, the top and 579 bottom edges outside the box plot represent the maximum and minimum values, respectively, 580 and the horizontal lines in the box plot represent the median of the set of data. The asterisk (*) 581 represents a significant difference from the control group.

582 Figure 4: Gene expression levels of CYP1A2 (A) and liver histological features $(\times 100)$ at 583 Day 30 after commencement of the control group (B) and treatment with $Q(C)$ and $L$ (D), 584 and EP-1 (E). The liver tissues in Figure B are visualized under the original magnifications of 585 586 hematoxylin and eosin staining.

\section{Tables:}

Table 1 Details of the real-time PCR primers used to detect expression levels of CYP1A2 Table 2 The number of fetuses among the four groups of voles at day 30 of the experiment

\section{Supplementary files:}

Table S1. Changes in body mass of voles in the three fertility control agent-treated and control groups.

Figure S1 The uterus morphology of the control and three sterility treatment groups at Day 30 after commencement of the treatment.

Figure S2 Histological features of the ovary $(A, \times 100 ; B \times 200)$ at Day 30 after commencement of the treatment with L and Q and EP-1.

Figure S3 The testicular morphology of the control and three sterility treatment groups at Day 
59930 after commencement of the treatment.

600 Figure S4 Histological features of the testis $(\times 100)$ at Day 30 after commencement of the

601 treatment with L and Q and EP-1. (A, control group; B, L group; C, Q group, D, EP-1 group). 602 
Figure 1

Figure 1: Body masschanges of voles in the three fertility control agent-treated groups andcontrol group.

The asterisk $(*)$ represents a significant difference from the control group.

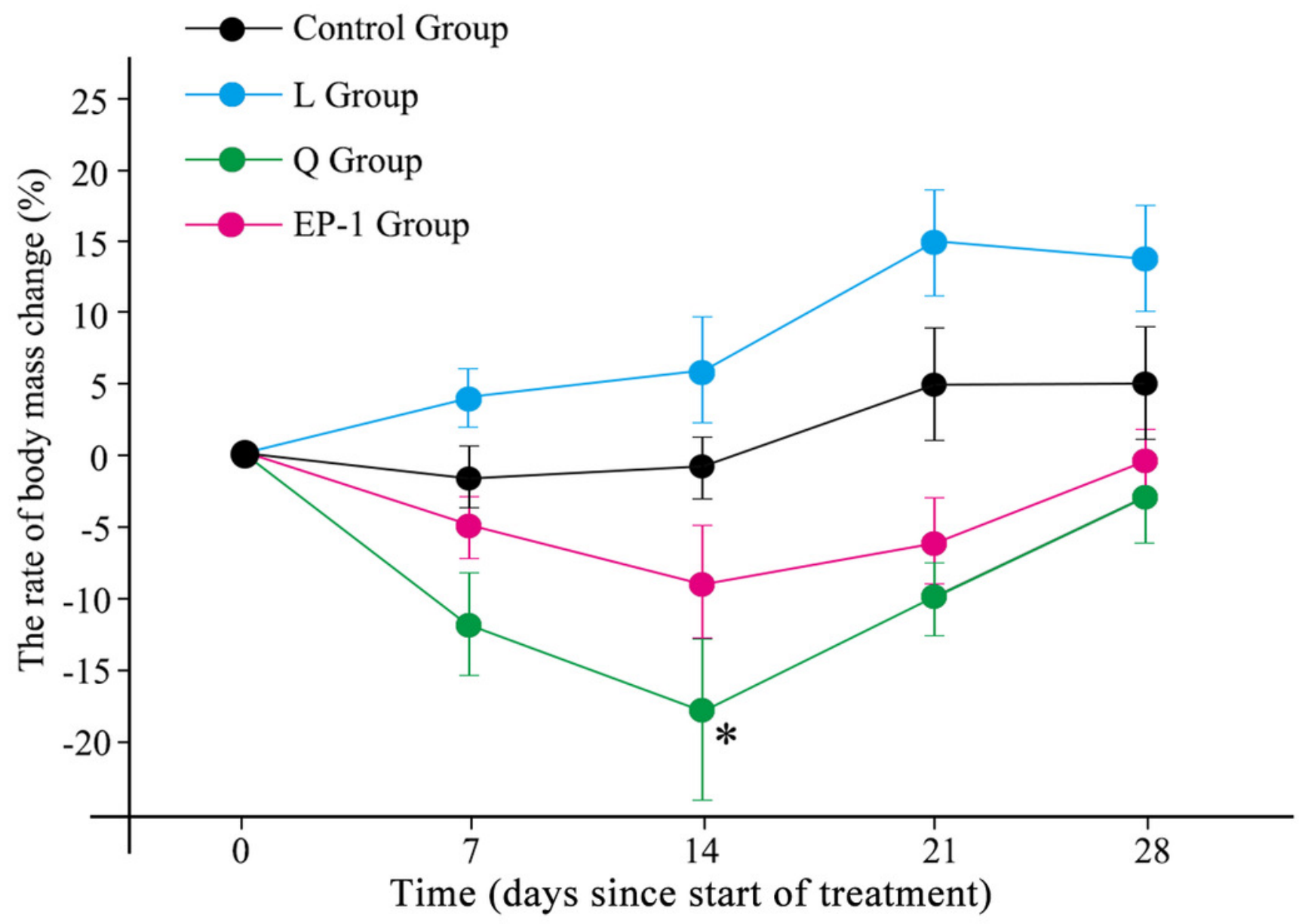




\section{Figure 2}

Figure2: Uterine weight (A), ovarian weight (B), testicular weight (C), and epididymial weight (D) in the four groups of voles at day30 of the experiment.

Values are presented as mean $\pm \mathrm{SE}$. The asterisk $(*)$ represents a significant difference from the control group.
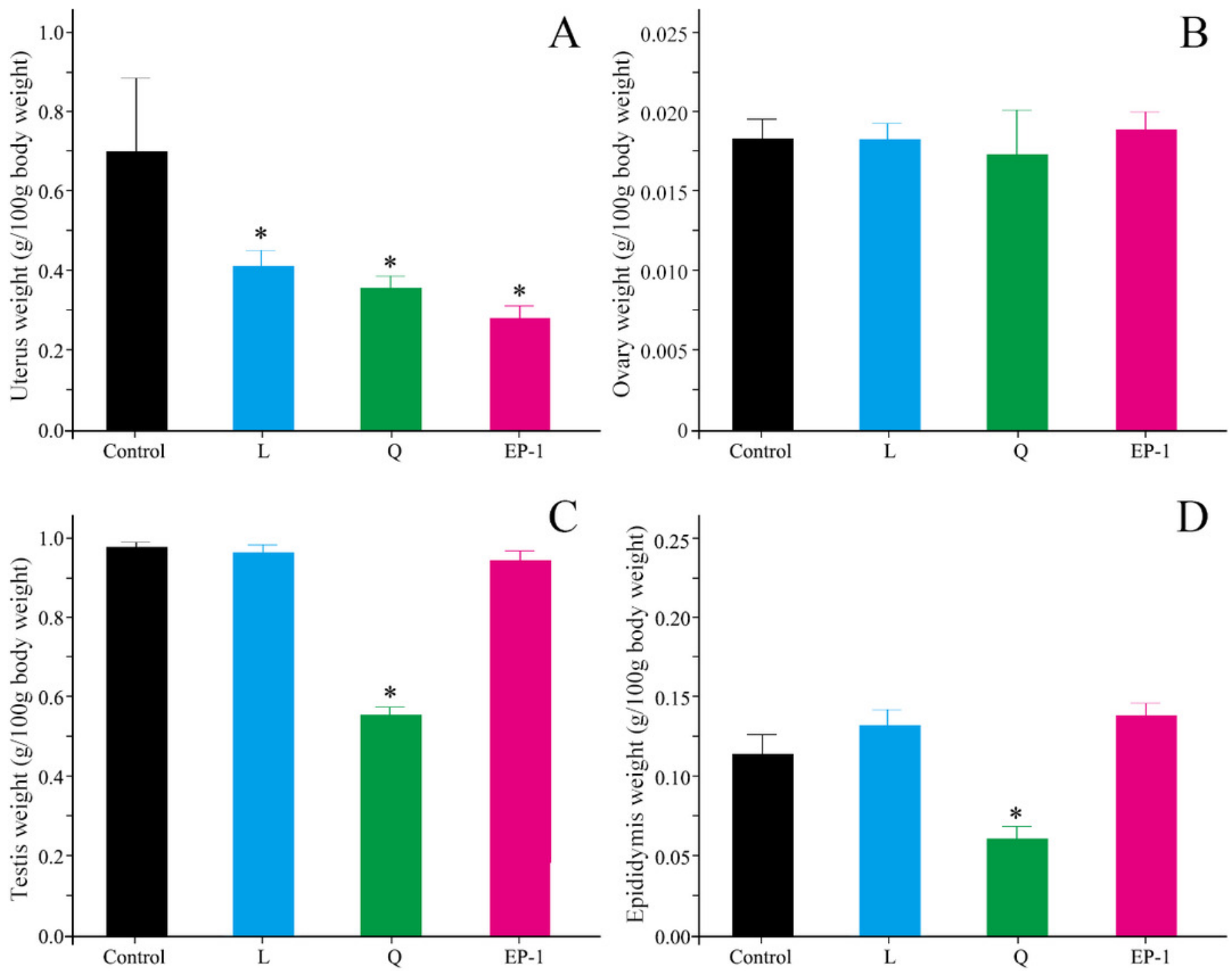
Figure 3

Figure 3: Levels ofestradiol (A), follicle-stimulating hormone (B), testosterone (C), and corticosterone (D) in the four groups ofvoles on day 30 of the experiment.

Estradiol and follicle-stimulating hormone were measured only in females, and testosterone and corticosterone were measured only in males. Each group of data is represented by a box plot. In the group of data, the top and bottom edges outside the box plot represent the maximum and minimum values, respectively, and the horizontal lines in the box plot represent the median of the set of data. The asterisk (*) represents a significant difference from the control group.
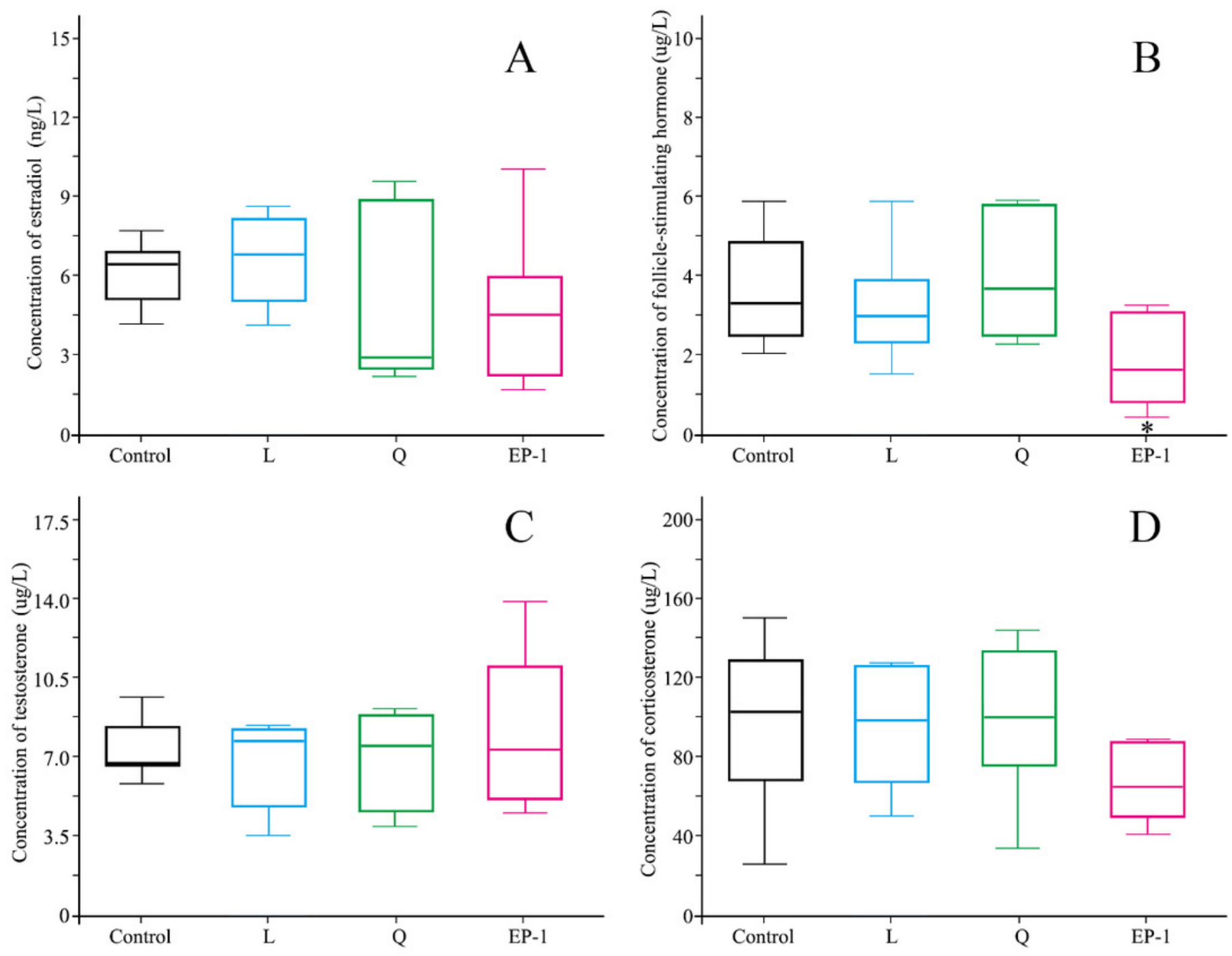


\section{Figure 4}

Figure 4: Gene expression levels of CYP1A2 $(A)$ and liver histological features $(\times 100)$ at day 30 after commencement of the control group (B) and treatment with $Q(C)$ and $L(D)$ and EP-1 (E).

The liver tissues in Figure B are visualized under the original magnifications of hematoxylin and eosin staining.
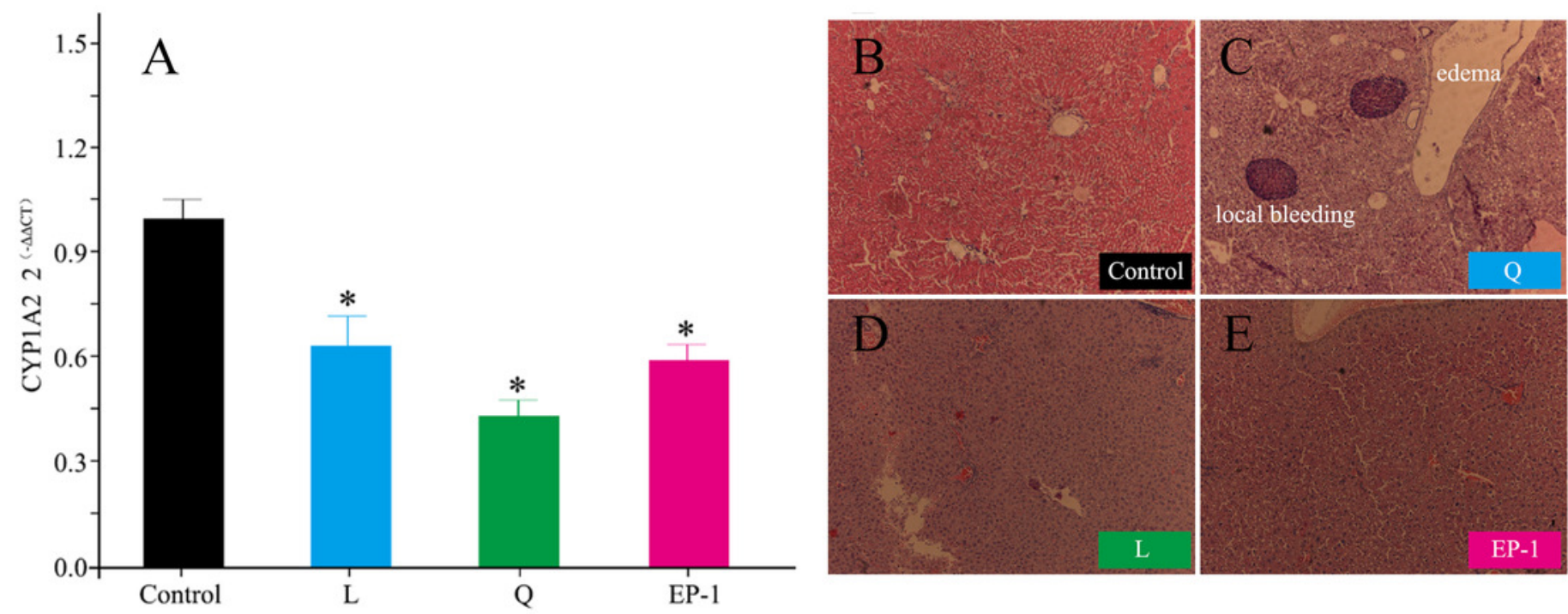


\section{Table $\mathbf{1}$ (on next page)}

Table 1 Details of the real-timePCR primers used to detect expression levels of CYP1A2. 
1 Table 1 Details of the real-time PCR primers used to detect expression levels of CYP1A2.

3

4

\begin{tabular}{lcccc}
\hline Gene name & Sequences & Length (bp) & Annealing temperature & PCR efficiency \\
\hline & Forward: TCGTCCTCTTGCTACTTA & & & \\
CYP1A2 & & $1266 \mathrm{bp}$ & $55^{\circ} \mathrm{C}$ & $0.91 \pm 0.02$ \\
& Reverse: TCGTCCTCTTGCTACTTA & & \\
& & & & \\
\end{tabular}




\section{Table 2 (on next page)}

Table 2 The number offetuses among the four groups of voles at day 30 of the experiment. 
1 Table 2 The number of fetuses among the four groups of voles at day 30 of the experiment.

\begin{tabular}{cccc}
\hline Group & Pairing number & Pregnancy rate $(\%)$ & Number fetuses per pregnant female \\
\hline Control & 7 & $85.71 \%(6)$ & $8,7,2,9,7,1$ \\
Quinestrol & 7 & 0 & 0 \\
Levonorgestrel & 7 & $14.29(1)$ & 2 \\
EP-1 & 7 & 0 & 0 \\
\hline
\end{tabular}

2 